\title{
Structuring and Presenting Lifelogs based on Location Data
}

\author{
Basel Kikhia ${ }^{1}$, Andrey Boytsov, Josef Hallberg, Zaheer ul Hussain Sani, \\ Håkan Jonsson, and Kåre Synnes \\ Luleå University of Technology \\ Department of Computer Science, Electrical and Space Engineering \\ 97187 Luleå, Sweden. Tel: 092049 10 00, Fax: 0920491399 \\ \{Basel.Kikhia, Andrey.Boytsov, Josef.Hallberg, Hakan.Jonsson, Kare.Synnes\} @ltu.se, \\ zaheer_sani@comsats.edu.pk
}

\begin{abstract}
Lifelogging techniques help individuals to log their life and retrieve important events, memories and experiences. Structuring lifelogs is a major challenge in lifelogging systems since the system should present the logs in a concise and meaningful way to the user. In this article the authors present a novel approach for structuring lifelogs as places and activities based on location data. The structured lifelogs are achieved using a combination of density-based clustering algorithms and convex hull construction to identify the places of interest. The periods of time where the user lingers at the same place are then identified as possible activities. In addition to structuring lifelogs the authors present an application in which images are associated to the structuring results and presented to the user for reviewing. The proposed approach allows automatic inference of information about significant places and activities, which generates structured image-annotated logs of everyday life.
\end{abstract}

Keywords: activity recognition, activity inference, lifelogging, clustering algorithms, SenseCam, GPS.

\section{Introduction}

Lifelogging is the act of digitally recording aspects and personal experiences of someone's life. Some people are interested in logging their life's activities for fun, medical purposes or diary applications [1]. It is important for many individuals to retrieve moments and events such as trips, weddings, concerts, etc. Reminiscing previous events among a group of people not only helps in remembering those events, but it also creates tighter social bonds and improves relationships among them [2]. Aiding memory is also one of the benefits that people gain by logging their life. For example, a lifelogging system can be used as an external memory aid that supports a person with memory problems by using reminiscence therapy [3]. In reminiscence therapy the user reviews and talks about the day with someone, such as a caregiver. The review and discussion act both as a social activity and as assistance for the user to remember. For this purpose, and to improve events retrieval using lifelogging in general, lifelogs need to be properly structured. Structuring lifelogs is the primary issue being addressed in this article.

A natural way to structure lifelogs is in the form of activities; for example having lunch, sitting in the park, shopping, attending a seminar, etc. This structuring requires techniques for reasoning and inferring of activities from the logged data. The logged data

\footnotetext{
${ }^{1}$ Corresponding author
} 
is part of the lifelogs and the granularity, as well as the types of data, can vary. However, the basic context should be captured to infer activities. This basic context have been analysed and identified as identity, location, activity and time, where locations and activities are of special importance [3] [4]. Context data could be captured by mobile devices carried by the user such as wearable sensors. It is good, however, to use a single mobile device when logging as the number of devices the user needs to carry should be kept to a minimum.

Just structuring data into activities based on context may not be sufficient for efficient retrieval and to support people reviewing their life experiences. Both context (e.g. time, locations and places) and content (e.g. images) need to be aggregated and segmented into the activities and be given semantic meaning. In previous work the authors have explored using known places to create this semantic meaning [3]. However, this approach is limited to predefined places. A desired solution would be finding places of importance and then inferring activities automatically. In this article the authors introduce an approach to detect new places and then infer activities automatically relying solely on time-stamped location data. Location and time are rich and easily accessible sources of context information that are relevant to find places of importance, where the user spent significant time. Being for a period of time in a significant place might be an indication of some activities happened in the place. The first problem that the article addresses is: "How can places of importance be recognized and activities be inferred based on location data and time?"

Once lifelogs are segmented into activities, they can be annotated with content, such as images and descriptions. Images play a vital role in enriching the logs and in supporting reminiscence processes in a lifelogging system [5] [6]. Images can be captured automatically by purpose-built devices (e.g. SenseCam which is further described in Section 5) or by a smart-phone carried in a way that allows it to capture images. However, the information and the images still need to be presented to the user in a way that takes advantage of the structured lifelogs. The second problem that this article addresses is: "How can structured lifelogs be presented so the user can review and retrieve the life experiences?"

The rest of this article presents the work done to address the problems listed in the introduction and is organized as follows: section 2 shows what algorithms have been used in this work to recognize new places. The calibration of the chosen place recognition algorithms is presented in section 3. Section 4 discusses the algorithm that has been used to infer activities. The development and deployment of the prototype application, which organizes the logs and presents them to the user, is the topic of section 5. Section 6 presents some of the related work and section 7 discusses the research questions. Finally, section 8 concludes the paper and presents the future work.

\section{Recognizing places of importance}

One of the problems addressed in this article is how to recognize places of importance for the purpose of structuring lifelogs. This is important because the places people visit contain hints towards the activities taking place. In fact, time-stamped location data can be used to recognize relevant places, and then infer activities based on identified places and time. In practice, areas where the user spends a significant amount of time can be 
seen as important locations or as activities done by the user at a specific location. One of the common approaches for discovering interesting patterns and data distributions from location data is density-based clustering algorithms [7] [8]. For instance, these algorithms can infer information of areas where the user spent significant time when having location data logged by a mobile sensor carried by the user [9] [10].

The proposed Place Recognition Algorithm relies on GPS points as a source of location data. The algorithm, however, is not restricted to GPS location information only. The adopted approach is depicted in Figure 1 and works as follows:

1. Raw time-stamped location data are used as an input for the approach.

2. Location data are clustered to identify places.

3. A convex hull is constructed over each cluster to estimate the geographical boundaries of the place.

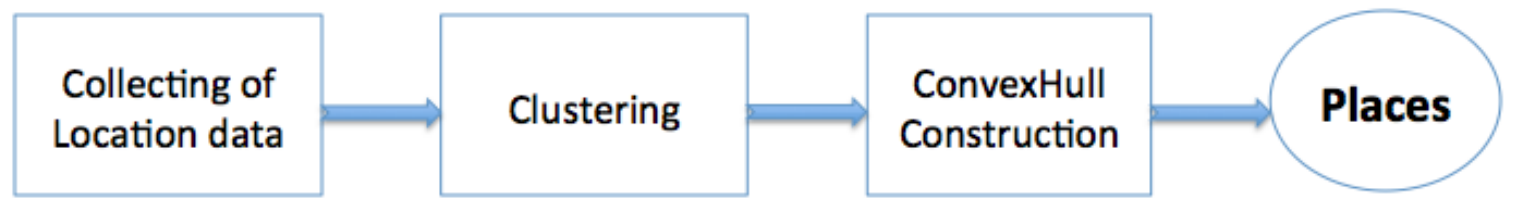

Figure 1. New Places Recognition - Action Flow

The aim of the clustering algorithm is to identify places of importance to the user, which are previously unknown in the system. These places can be confirmed and labelled by the user while reviewing the lifelogs. If the user confirms a place, the system will add the coordinates that correspond to this place and define the place as a known one. The algorithm compares each GPS point with all previously known places. If the point belongs to a known place, the algorithm will remove it from the input set, but keep it for inferring activities later on. If the point does not belong to a known place, the algorithm will keep it in the input set for clustering. The GPS points in the input set are then clustered and aggregated regardless of time. Such clusters are signs of places where the user spent significant time. For example, the user might go to the office at different times of the day but the place is still the same.

The following requirements should be taken into consideration when choosing the clustering algorithm:

- The number of clusters is initially unknown. Different users have different numbers of visited places. The user can also visit a place that is never visited before. Algorithms which have pre-defined number of clusters will consequently not work in that case.

- Some GPS points might not correspond to a significant place for the user. For instance, the user might walk home from the working place. The location data, captured on the way home, do not belong to a significant place. The clustering algorithm should therefore allow a point not to be a part of any cluster.

- The algorithm should be noise-tolerant and resistant to outliers. Even though GPS points correspond to the user's position, outliers can appear when the user is inside a 
building when there are not enough satellites detected. These outliers should be identified as noise.

Density-based clustering algorithms satisfy all the aforementioned requirements. Two algorithms of density-based clustering approach were used by the authors: Density-Based Spatial Clustering of Applications with Noise (DBSCAN) [7] and Ordering Points To Identify the Clustering Structure (OPTICS) [8]. OPTICS can be viewed as extension of DBSCAN. While DBSCAN provides faster runtime clustering, OPTICS supplies the developers with additional analysis tools and better visualization of the results [8]. Therefore, DBSCAN was implemented to cluster location data and then OPTICS-based analysis was used for algorithm configuration and parameters adjustment.

After the clusters are identified, the system constructs the convex hulls to estimate the geographical boundaries of the places. The convex hull of a set of points $Q$ is the smallest convex polygon $P$ for which each point of $Q$ is either on the boundary of $P$ or in its interior [11]. It is usually assumed that points in the set $Q$ are unique and that $Q$ contains at least three points that are not collinear. Rubber band analogy is often used for better understanding. Each point in $Q$ is considered as being a nail sticking out from a board. The convex hull is then the shape formed by a tight rubber band that surrounds all the nails [11]. Figure 2 illustrates the view of the place clusters after implementing DBSCAN over the location data and constructing the convex hull.

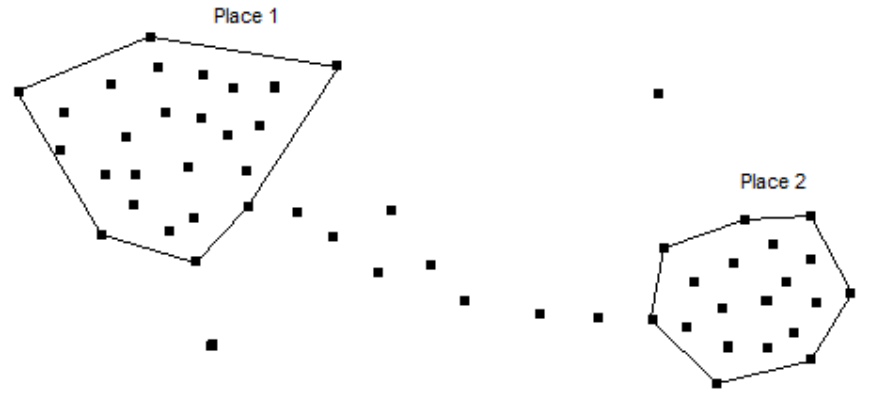

Figure 2. Recognized places

\section{Calibrating the Place Recognition Algorithm}

Algorithms like the aforementioned density-based clustering algorithm have parameters that should be set in advance. Therefore, the parameters' values should be calibrated and tuned so the algorithm produces a minimum number of errors.

DBSCAN algorithm uses two parameters: the Radius, the range around a point where other points in that range are considered neighbours, and MinPts, minimum number of neighbours that a point needs in order to not be declared as noise. After setting the parameters, the algorithm forms clusters using the density of local neighbourhoods of points. This is done by selecting a point and then assigning all the points within its Radius (high destiny neighbours) to the same cluster. This approach is repeated for all the points resulting in many clusters with different arbitrary shapes. All points that do not belong to any cluster are considered noise.

A suitable set of parameters is the set that results in fewer numbers of place recognition errors. In order to evaluate the number of place recognition errors that correspond to 
different parameter sets, real-life data were collected and labelled manually, the possible error types were defined and then the performance of different parameter sets was estimated with respect to the identified error types.

\subsection{Data Collection}

A Windows Mobile application has been developed to log GPS tracks periodically every 30 seconds. If there are no satellites detected when logging, the application will do nothing and wait another 30 seconds to look for satellites. When connecting the logging device to a computer, the application transfers the logs as an XML file that contains longitude, latitude, logging time, speed, and number of satellites. The application then deletes the logs from the logging device so they do not interfere with new logs. Three users have done the data collection over a period of six months. The users were asked to carry a mobile device, with the application installed, during the day. By the end of the day, the user connects the mobile to a computer to transfer the logs.

Relying on time-stamped GPS data has some limitations. Firstly, GPS data might be noisy and not accurate if few satellites were detected when logging the location. To overcome this limitation, the logged GPS data with less than 4 satellites are ignored. Having 4 satellites or more showed good results when doing manual analysis. Blocking the view of the GPS receiver by another object is another limitation. This happens sometimes when the user has keys, for instance, together with the mobile device in the same pocket. This case results in missing some location data or having noisy data. During the data collection period, the users were advised to not have any other object next to the mobile device to reduce the risk of blocking the access to satellites.

The users were also involved in the evaluation process to ensure that the algorithm's results correspond to significant places.

\subsection{Error Types}

Manual analysis of the collected data revealed that there are 4 types of possible errors:

1. The algorithm detected a cluster that does not correspond to any real-life place. The impact of this error is the least severe of all four. It is possible for the user to manually fix this by discarding this cluster when reviewing the lifelogs. This kind of error can also result in detecting non-existent activities on later activity inference steps, but this can be also fixed when reviewing the logs. However, this problem can still result in distraction and waste time for the user. The amount of errors of this kind tends to grow with increasing the Radius or with decreasing MinPts.

2. The algorithm merged two places into one. This problem causes distraction to the user and it can disrupt the activity inference process. The number of errors of this kind tends to grow with increasing the Radius or with decreasing MinPts.

3. The algorithm separates one place into two different ones. This problem is opposite to the previous one. The number of errors of this kind tends to grow with decreasing the Radius or with increasing MinPts.

4. The algorithm did not detect an essential place. This error type is the most serious one because the activities in that place will be lost as well. The number of errors of this kind tends to grow with decreasing the Radius or with increasing MinPts. 


\subsection{Parameter Values}

25 randomly chosen logs were analysed to determine the best parameter values of the DBSCAN algorithm. Each log has data collected during one day. Logs were manually analysed and essential places were identified based on observation.

The DBSCAN algorithm has been implemented using JavaScript and the results have been shown through a web application and manually processed to identify errors of different types. The application shows a map with all collected points during the day on the left side, and the clustering results after applying DBSCAN based on the Radius and MinPts on the right side. Figure 3 presents part of the results when running DBSCAN on one selected $\log$ with 20 meters as a Radius and 3 points as MinPts. The points that are marked by 1 belong to one cluster while the points that are marked by 2 belong to another cluster.

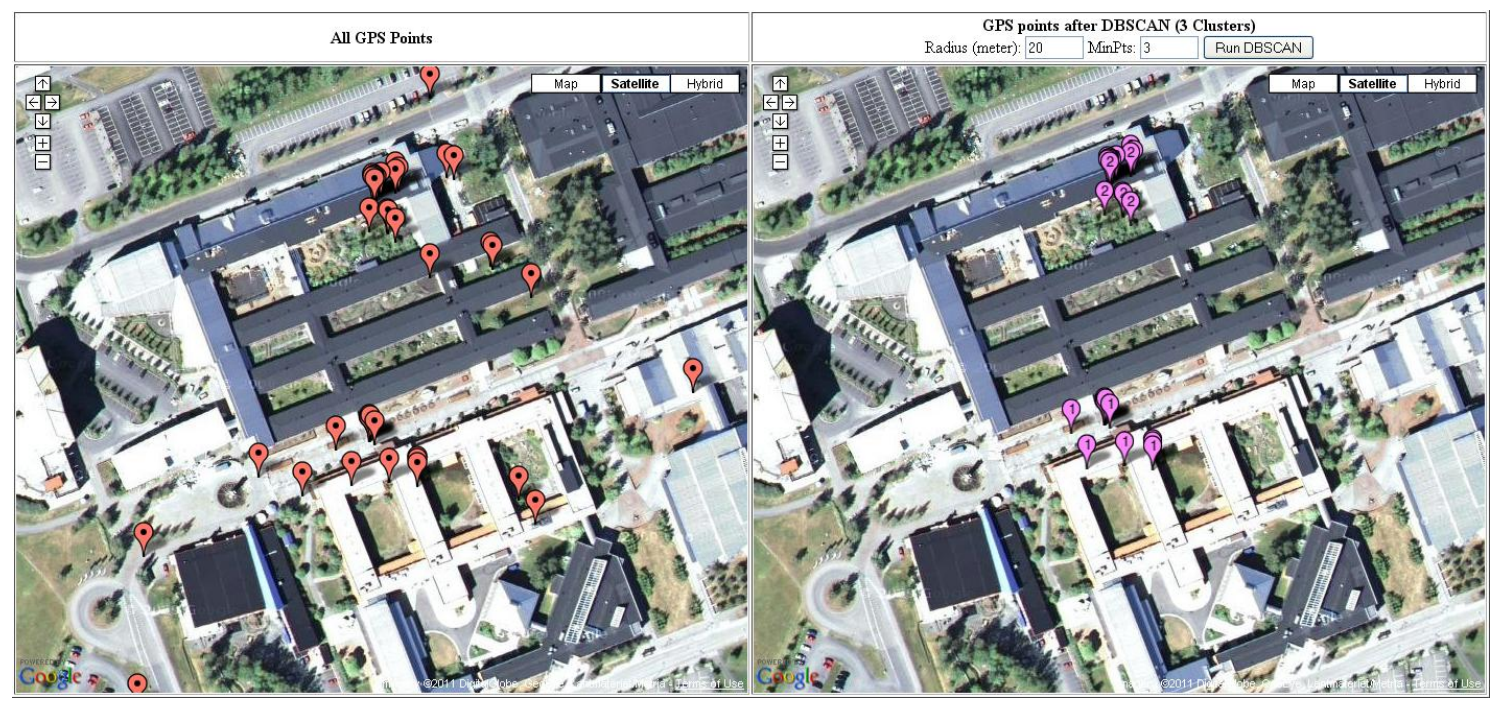

Figure 3. DBSCAN implemented in a web application

Different reasonable values of the Radius and MinPts were tested to find out what errors they produce. For each $\log$, the following parameters sets were considered: every possible MinPts from 2 to 20 with the step of 1, combined with every possible Radius from 5 meters to 30 meters with the step of 5. The aforementioned trends of errors (error types in section 3.2) allowed finding the best values without checking all the combinations of every MinPts with every Radius. Those heuristics significantly reduce the number of parameter combinations for manual testing, and practically allow to check just $\sim 10-30$ parameter combinations per log. The aim is to find the minimum values of MinPts and the Radius that result in fewer numbers of errors for each log. The priority is to reduce the errors of type 4 when the algorithm does not detect essential places. After determining the best values for each log, the average of those values is calculated to find out a representative value for the whole set of logs. Table 1 shows the best combination for minimum values of the Radius and MinPts for each log followed by the average values. 


\begin{tabular}{|c|c|c|}
\hline GPS Log\# & MinPts & Radius (meters) \\
\hline 1 & 2 & 10 \\
\hline 2 & 4 & 10 \\
\hline 3 & 2 & 15 \\
\hline 4 & 3 & 10 \\
\hline 5 & 3 & 5 \\
\hline 6 & 2 & 10 \\
\hline 7 & 3 & 30 \\
\hline 8 & 4 & 5 \\
\hline 9 & 3 & 5 \\
\hline 10 & 5 & 10 \\
\hline 11 & 2 & 5 \\
\hline 12 & 3 & 30 \\
\hline 13 & 2 & 20 \\
\hline 14 & 2 & 5 \\
\hline 15 & 4 & 15 \\
\hline 16 & 3 & 10 \\
\hline 17 & 3 & 5 \\
\hline 18 & 2 & 15 \\
\hline 19 & 3 & 10 \\
\hline 20 & 6 & 20 \\
\hline 21 & 4 & 5 \\
\hline 22 & 3 & 15 \\
\hline 23 & 2 & 10 \\
\hline 24 & 5 & 25 \\
\hline 25 & 6 & 20 \\
\hline Average & 3.24 & 12.8 \\
\hline Tat & $347 m a r a t$ & 9 \\
\hline 19 & 3 & 1095 \\
\hline
\end{tabular}

Table 1. Summarization of the logs analyses

The average value for MinPts is 3.24, while it is 12.8 for the Radius. Since increasing MinPts might result in increasing the number of undetected places, the value 3.24 is rounded to 3 . Thus the parameter values that yield the best results for the DBSCAN algorithm are: 3 for MinPts and 12.8 meters for the Radius.

As an additional method of parameters adjustment the OPTICS algorithm was used [8]. OPTICS operates with the same parameters as DBSCAN, but while MinPts should be specified manually, the neighbourhood radius can be adjusted by the analysis of reachability plots. The plots visualize the structure of clusters and sub-clusters. Analysis of OPTICS reachability plots can confirm the values of the DBSCAN parameters. The analysis also gives an indication of how stable the clustering approach is. To perform OPTICS-based analysis, the WEKA toolkit was used [12]. WEKA is a machine learning software that has a collection of visualization tools and algorithms for data analysis and predictive modelling.

Two sample reachability plots are depicted in figure 4. Reachability plots (a) and (b) correspond to two different GPS logs chosen out of the $25 \operatorname{logs}$ of testing. MinPts is set to 3 and the green threshold lines correspond to the Radius value of 12.8 meters. The plot areas below the threshold line correspond to clusters. In the reachability plot (a) there are 
8 clusters, while there are 2 clusters in the reachability plot (b). This visualization can identify how significant change of the neighbourhood radius is required to obtain different clustering results. For example, the leftmost cluster of the figure 4 (b) would have been recognized as 3 separate clusters if the neighbourhood radius is set below 10 meters. If the reachability plots contain many values near the threshold, it means that the clustering is unstable, and the number and the structure of the clusters can be heavily influenced by random fluctuations in GPS measurements. The analysis of reachability plots allowed the authors to conclude that for the vast majority of the testing samples it takes significant change of the neighbourhood radius to alter the clustering results. Therefore, the chosen parameter values provide stable clustering and low sensitivity to random factors.

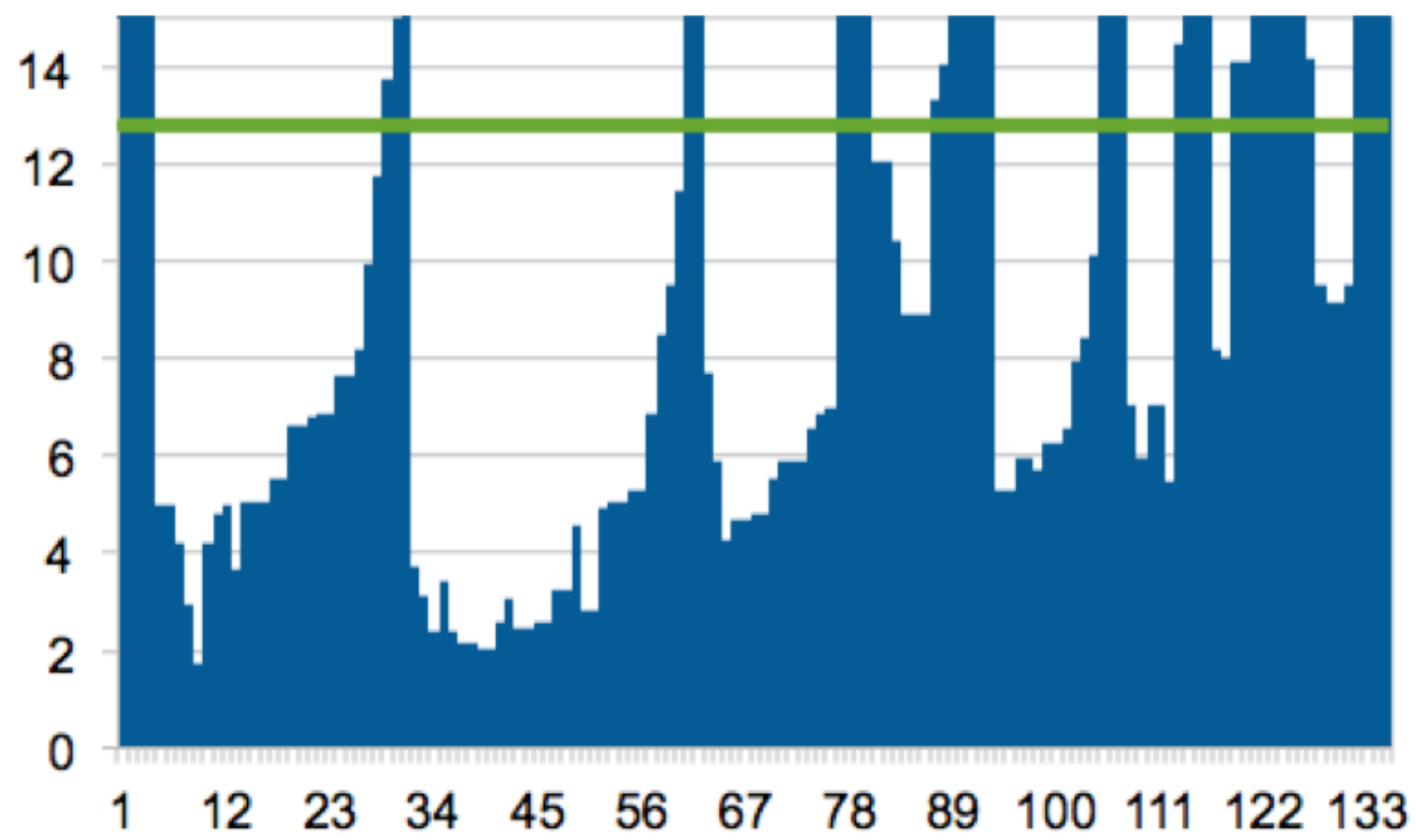

Reachability Plot (a). The green line represents the threshold value of the Radius 12.8 meters. 8 clusters are recognized (the white areas below the threshold) 


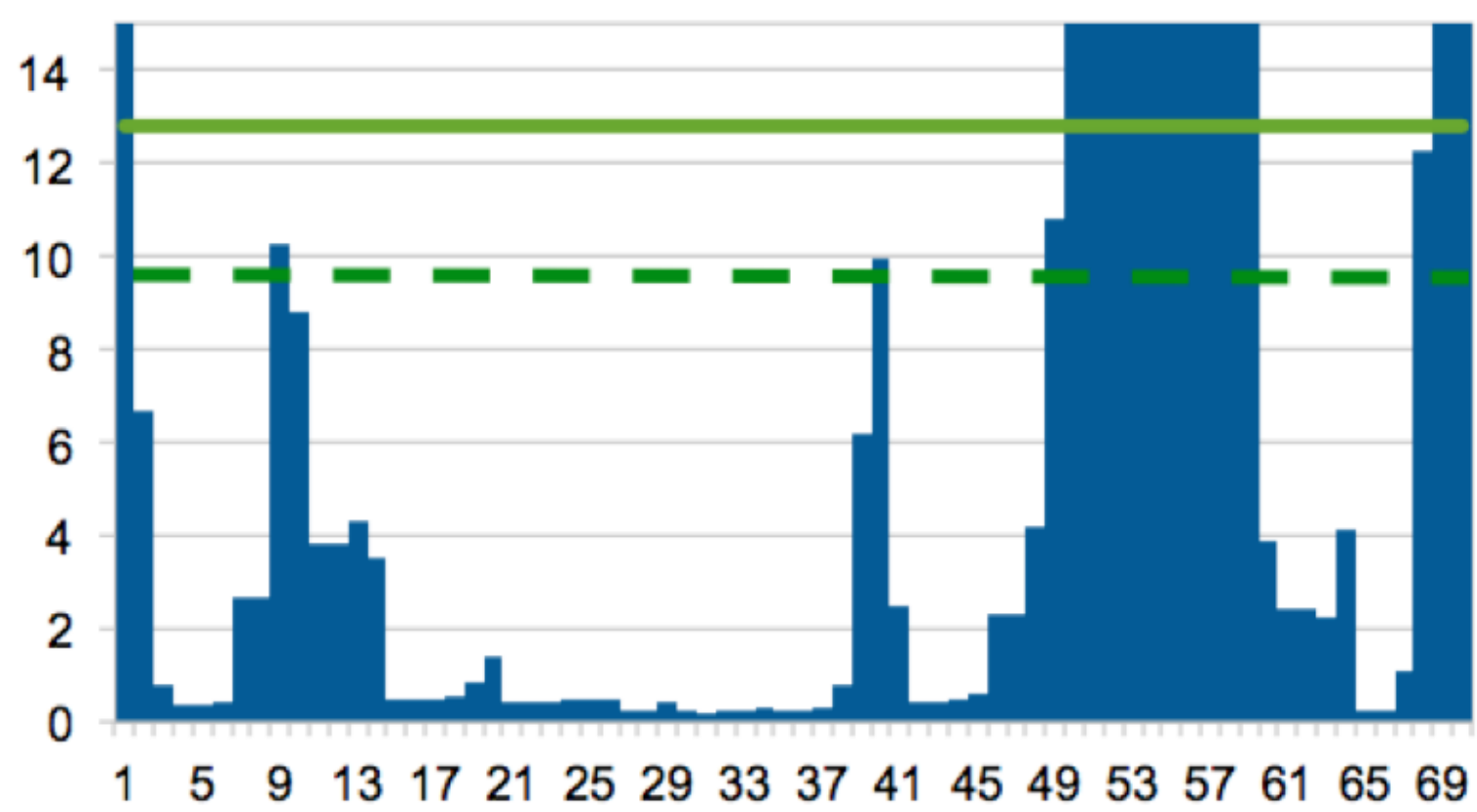

Reachability Plot (b). The green dashed line represents the threshold value of the Radius if it is changed from 12.8 meters to less than 10 meters. The leftmost cluster will be recognized as 3 clusters.

Figure 4. Reachability Plot visualization when using OPTICS

\section{Inferring Activities}

Once places of importance have been identified then they can be used to infer activities.

To reason about activities, the following properties of an activity are identified:

1. An activity occurs at a place. The place can be a new one, which was just obtained by the place recognition algorithm, or a previously known one for which the geographical boundaries are known. This property leads to several important consequences:

- The GPS points that correspond to the same activity are defined as a subset of the GPS points that correspond to the same place. This reduces the scope of the algorithm from the entire set of GPS points to just points within places.

- If the user leaves a place, even for a short time, the activity is interrupted. This consequence can help in distinguishing several activities that happened in the same place at different times.

2. An activity takes a certain amount of time.

Based on these properties, a set of GPS points is an indication of an activity if:

- The points belong to the same place.

- The points are sequential in time.

The main idea of the activity inference algorithm is to decompose all place clusters into sub-clusters that do not overlap with each other in time. Place cluster refers to the set of GPS points within the place. Overlapping occurs when the user leaves place $A$, for example, to place $B$ then comes back later to place $A$. For instance, the user spends time 
in the office from $8 \mathrm{AM}$ to $1 \mathrm{PM}$, goes to a restaurant for lunch from $1 \mathrm{PM}$ to $2 \mathrm{PM}$, back to the office from $2 \mathrm{PM}$ to $5 \mathrm{PM}$ and finally goes to the same restaurant from $5 \mathrm{PM}$ to 7 PM for dinner. The timeframe of the GPS points captured when being in the office will be from $8 \mathrm{AM}$ to $5 \mathrm{PM}$ while the ones captured when being in the restaurant will be from $1 \mathrm{PM}$ to $7 \mathrm{PM}$. Decomposing the office cluster and the restaurant cluster into sub-clusters that do not overlap in time will result in 4 sub-clusters that represent 4 different activities:

- From 8 AM to 1 PM (being in the office)

- From 1 PM to 2 PM (being in the restaurant)

- From 2 PM to 5 PM (being in the office)

- From 5 PM to 7 PM (being in the restaurant)

To identify activities that happened at certain places based on the definitions in this section, the following algorithm has been introduced. For any place $A$, the earliest captured GPS point at this place is marked as A.begins, and the latest captured GPS point at this place is marked as A.ends. The fact that GPS points are naturally ordered by time makes calculating the timeframe easy. Clusters that correspond to the visited places are added to the input set of the activity inference algorithm.

\section{Algorithm:}

1. Take the first element of the input set (and remove it from the set). Let it be cluster A.

2. Search through the entire remaining parts of the input set. Search goes on until we find another element whose timeframe overlaps with the timeframe of A. Let it be cluster B.

3. If no overlapping cluster can be found:

3.1. Add cluster $A$ to the result set.

3.2. If the input set is not empty, go to step 1.

3.3. If the input set is empty, go to step 5.

4. If an overlapping cluster can be found:

4.1. Remove cluster $B$ from the input set.

4.2. Order the following points in time in ascending order: A.begins, B.begins, A.ends,

B.ends. Let's refer to the ordered time variables as time $1 \leq$ time $2 \leq$ time $3 \leq$ time 4 .

4.3. Add to the input set the following sub-clusters of cluster A:

1) Sub-cluster within timeframe [time1, time2]

2) Sub-cluster within timeframe [time2, time3]

3) Sub-cluster within timeframe [time3, time4]

4.4. Add to the input set the following sub-clusters of cluster B:

1) Sub-cluster within timeframe [time1, time2]

2) Sub-cluster within timeframe [time2, time3]

3) Sub-cluster within timeframe [time3, time4]

4.5. If the input set is not empty, go to step 1. 
5. End the algorithm and return the result set.

Output: a set of sub-clusters that do not overlap in time, each sub-cluster represents an activity.

The final timeframe of an activity can be obtained in a straightforward manner after the GPS points are attributed to activities; the time between the earliest point and the latest point of the activity. If a sub-cluster contains only one GPS point (lets refer to this only point as $P$ ), then the timeframe for this activity is counted as follows:

- If the sub-cluster is the earliest one, the timeframe of the activity is counted as the time between $P$ and the next collected point after $P$.

- If the sub-cluster is the latest one, the timeframe of the activity is counted as the time between $P$ and the previous collected point before $P$.

- If the sub-cluster is not the earliest or the latest one, the timeframe of the activity is counted as the time between the previous collected point before $P$ and the next collected point after $P$.

Figure 5 illustrates the view of activities after decomposing Place 1 (which is shown in figure 2) to sub-clusters that represent activities.
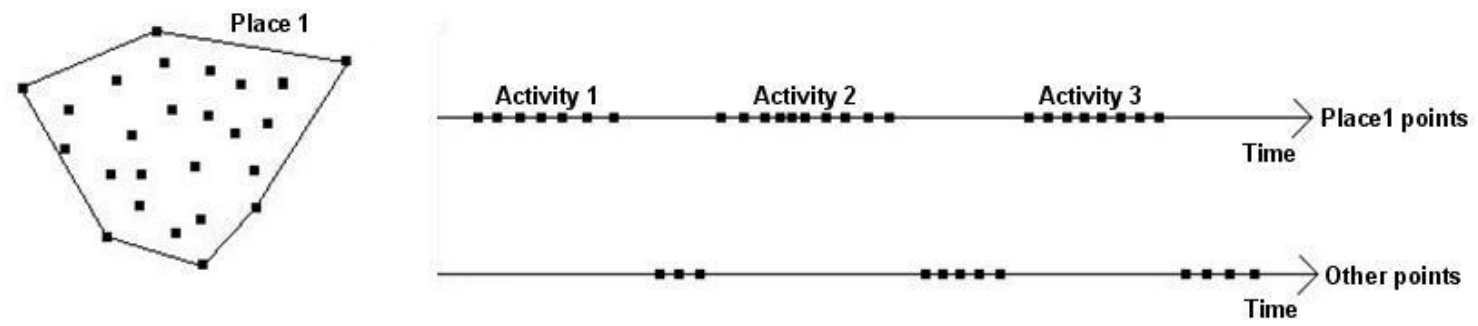

Figure 5. Recognized activities within a place

\section{Implementation and Deployment}

For lifelogs to be useful they need to be structured and presented to the user in a way that will give a good overview of content and data. The proposed solution for structuring lifelogs is to identify places of importance, infer activities, and then associate images with the places and the activities. A prototype application to demonstrate how this could be done, used with six months' worth of captured lifelog data, is therefore presented in this section.

The prototype consists of mobile devices for capturing images and contexts, and an application for reviewing the gathered data. The mobile device for capturing images that is being used in this prototype is called SenseCam, which is depicted in Figure 6. 


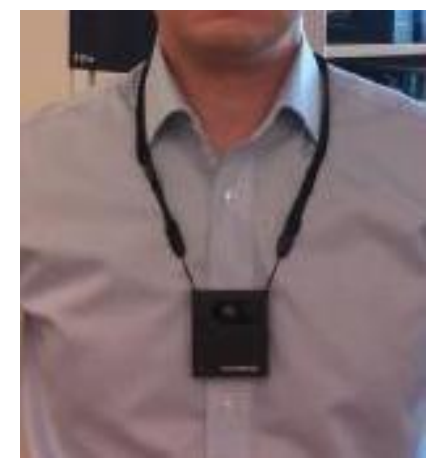

Figure 6. SenseCam worn around the neck

SenseCam is a wearable digital camera, which keeps a digital record of the activities that the person experiences [13] [14] [15] [16]. All recordings are automatically logged without the user's intervention and therefore without any conscious effort [14]. SenseCam contains a number of different electronic sensors which can be used to collect data for the lifelogs: light-intensity and light-color sensors, a passive infrared (body heat) detector, a temperature sensor, and a multiple-axis accelerometer. Certain changes in sensor readings can also be used to automatically trigger a photograph to be taken [17] which helps capturing things of significant importance. SenseCam does not feature a GPS sensor, so location data was instead captured by a smartphone, synchronized with the SenseCam.

When connecting the two devices, the SenseCam and the smartphone, to a computer with the prototype application installed, the system performs the following steps:

1. Transferring the logs in the form of XML. The logs consist of time-stamped GPS data and time-stamped images.

2. Analyzing the GPS data to identify periods of time where the user visited known places during the day.

3. GPS points that do not correspond to any of the known places are aggregated, using the DBSCAN algorithm, into clusters that represent new places. The Radius is set to 12.8 meters and MinPts is set to 3 .

4. Inferring activities based on the places using the method presented in section 4.

5. Associating SenseCam images with the recognized places and the inferred activities based on time.

6. Showing the results on the main interface in a chronological order.

The prototype application associates SenseCam images with auto-recognized places and inferred activities. The middle image of each group of images that belongs to a place or to an activity is chosen as a representative image. When reviewing, the application shows not only the images, associated with places and activities as content, but also the location context data. This can improve the reviewing process as it combines context with content data. Figure 7 shows the main interface of the application after transferring the logs of one day. This interface consists of 2 columns, where one column presents places and the other one presents activities. 


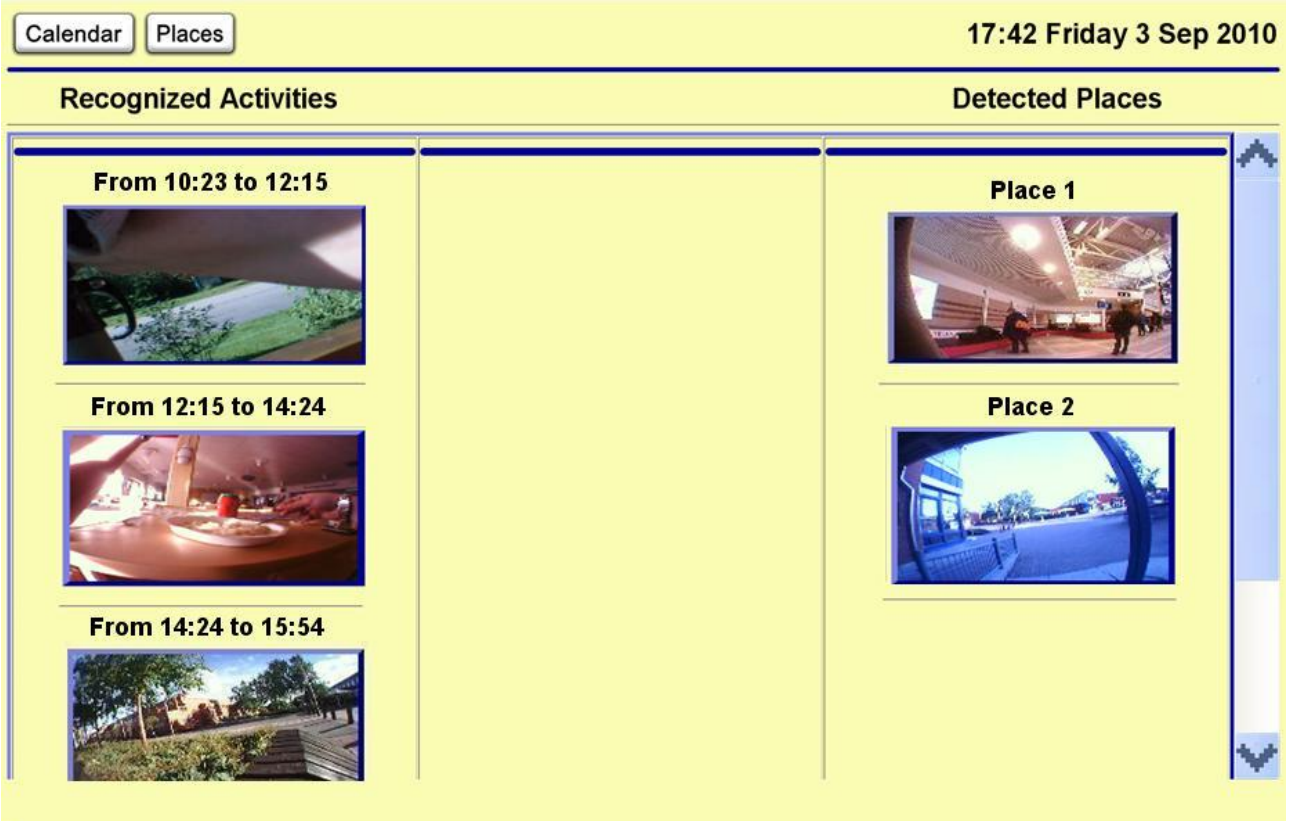

Figure 7. The main interface of the lifelogging application

\subsection{Reviewing Places}

Figure 8 shows the place page when reviewing. When reviewing a place, the system shows the constructed convex hull from the GPS points that correspond to the place. In addition, SenseCam images that have been captured when the user was in the place are shown.

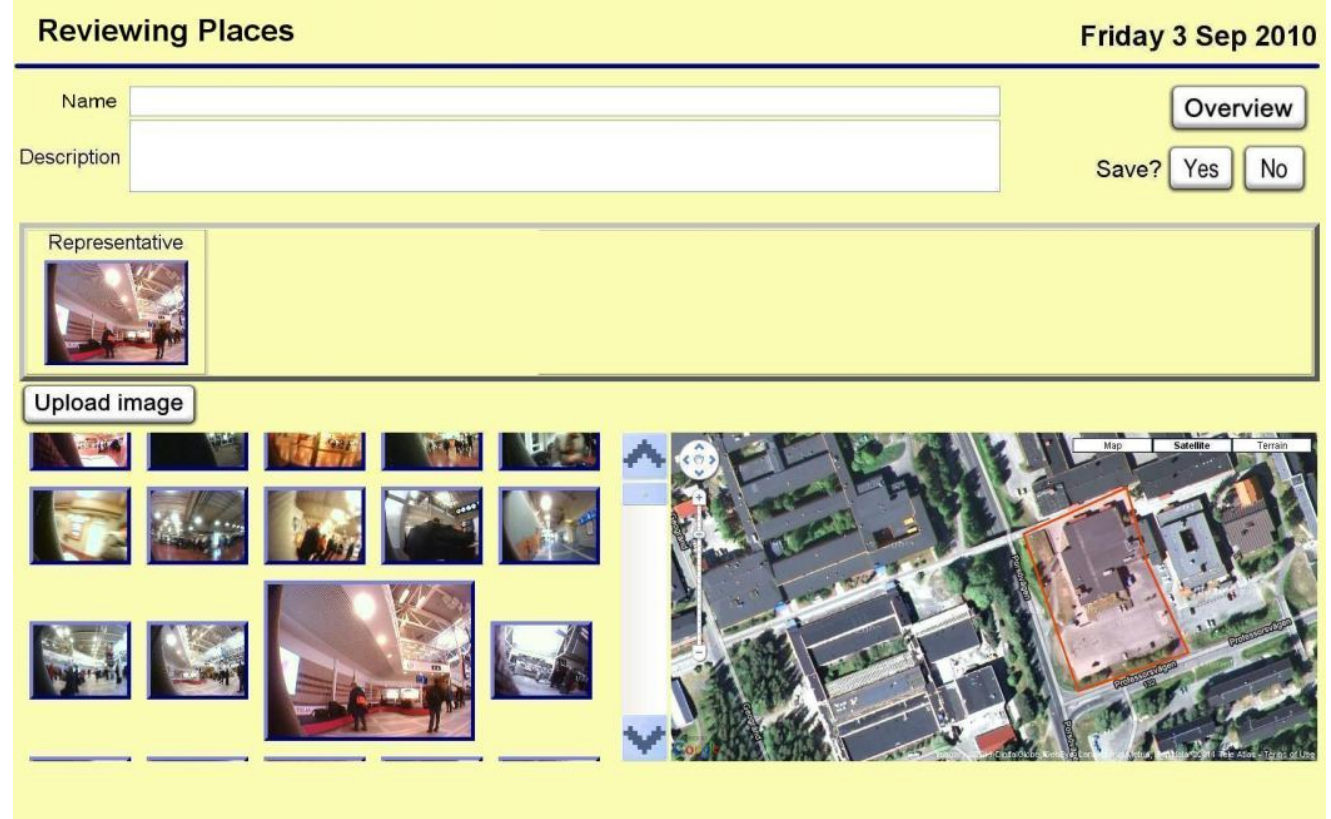

Figure 8. Reviewing a place within the lifelogging application

The user can choose a representative image for the place using the available SenseCam images. The user can also upload own images from an external source. If the user 
confirms the place, the system will save the chosen image as the representative one together with the coordinates that correspond to this place. Thus the place will be known and detected automatically by the system if the user visits it again. As the user continues to review the data, more places will be added to the list of known places of importance. This will improve the system's knowledge of important places, which will increase the level of automation in detecting the user's movements.

\subsection{Reviewing Activities}

When reviewing an activity, the system presents all SenseCam images that have been captured during that activity. The system also shows all the GPS points that correspond in time to this activity on a map. It is possible for the user to choose certain images to associate with the activity among the whole set of images. Figure 9 shows the activity page when reviewing.

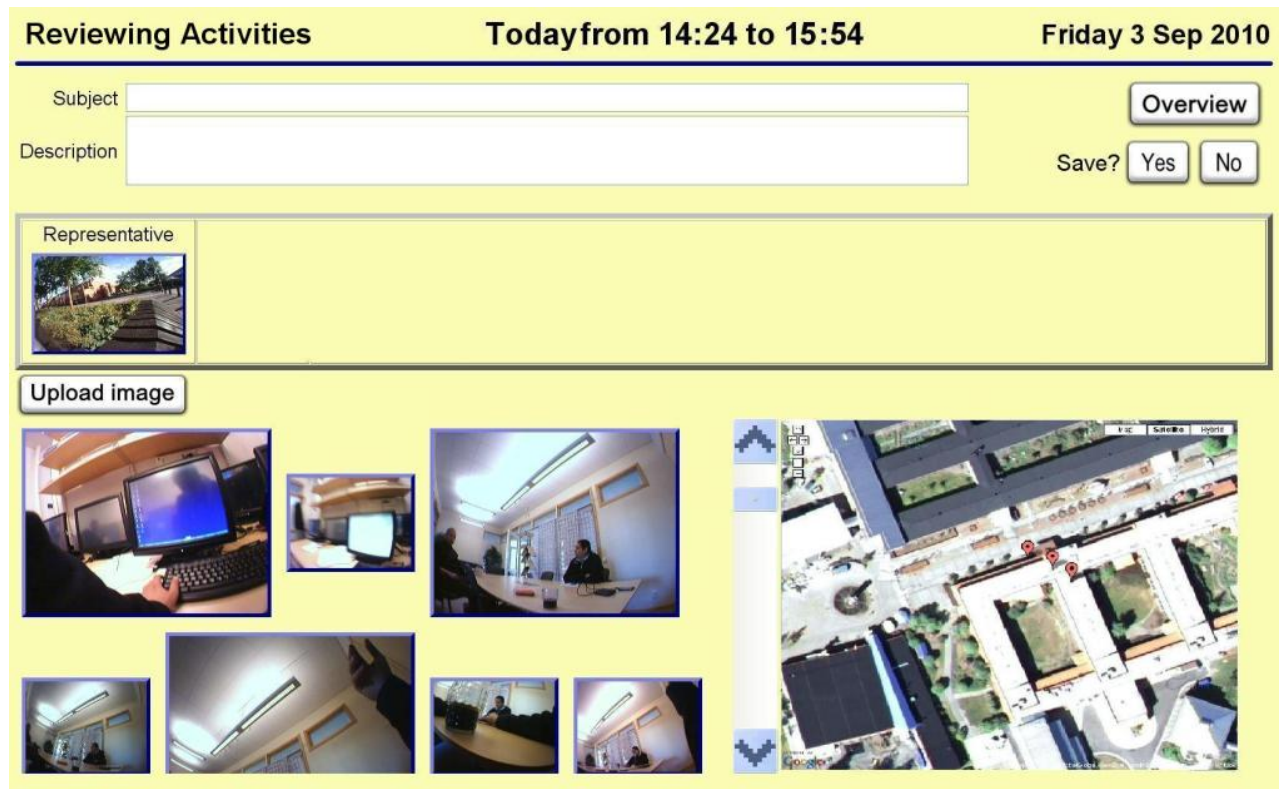

Figure 9. Reviewing an activity within the lifelogging application

Reviewing activities differs from reviewing places in the following aspects:

- It is possible to annotate an activity with many images, while a place can be annotated only with 1 image.

- The coordinates that correspond to an activity will not be saved in the system if the user chooses to save the activity.

- All the activities that happened in a place will be associated with this place for later context-dependent retrieval.

Saved activities can be retrieved later based on date/time or places. This means that the user can recall all the activities that happened at a certain time or in a certain place.

\section{Related work}

In this article the authors have shown how to recognize places of importance, how to use them to structure lifelogs in the form of activities, and how to present lifelogs to the user 
in a structured way. This section describes some of the work that has been done by others, which is related to the work presented in this article.

The DBSCAN algorithm, together with OPTICS, was used by the authors when clustering the location data. Another common clustering approach is K-means. K-means is a method of cluster analysis that aims to partition $n$ observations into $k$ clusters [18]. This method assigns $k$ initial means randomly, and then it goes over all the observations and assigns them with the nearest mean, which results in having $k$ clusters. The mean value of each cluster is calculated, and then the method is repeated until the error term is deemed small or the decrease is below a threshold. This method is very sensitive to noise, and the number of the clusters must be known in advance. K-means also rely on the random initialization of the means that makes it non-deterministic [19]. Ashbrook et al. [20] used a variant of the $\mathrm{k}$-means clustering algorithm that used GPS data in defining locations of the user. All the points within a pre-defined Radius are marked, and the mean of these points is computed. The system will do calculation again based on the mean and the given Radius to find a new mean value. When the mean value is not changing any more then all points within its Radius are placed in a cluster and removed from consideration. This approach is repeated until there are no more GPS points left. The main limitation of this approach is that the Radius should be set in advance and then the clustering algorithm will rely on that value. Density-based algorithms overcome the limitations of the K-means clustering method [7]. The advantages of using DBSCAN over K-means are mentioned by Zhou et al. [19]: DBSCAN is less sensitive to noise, it allows clusters of arbitrary shape, and it provides deterministic results.

Alvares et al. [21] presented an approach to add semantic information to trajectories. Trajectories are decomposed into stops and moves where a stop is a set of points that is transformed into a geographic object with a meaning, and a move is a small part of a trajectory between two stops. Candidate stops are identified in advance and the output of the work was a semantic trajectory dataset. Palma et al. [9] extended the work presented in the paper [21]. Palma et al. used a variation of the DBSCAN algorithm to discover interesting places in trajectories, which are previously unknown. Trajectories are observed as a set of stops and moves, where stops are more important. The authors in [9] calculated the distance between points along the trajectory instead of using Euclidean distance, and they used minimal time instead of using minimal number of points MinPts, for a region to be dense. The absolute distance (Eps) is used to calculate the neighbourhood of a point. The choice of Eps requires knowing the percentage of location points, which corresponds to stops. In our work the choice of Eps is based on analysis and observation of real-life data. In contrast to [21] and [9], our work considers activity inference and contains the methods to visualize and label the found places and activities.

Andrienko et al. [10] defined the trajectory of an object as temporally ordered position records of that object. The authors in [10] looked at the characteristics of the movement such as instant speed, direction, travelled distance, displacement and the temporal distances to the starts and ends of the trajectories. These characteristics are then represented as dynamic attributes that can give an indication of movement events. For instance, having low speed in some areas can be an indication of specific events belonging to those areas. The events are clustered according to their positions and time, and then used to extract places. Repeated occurrences of events in places are calculated 
by means of density-based clustering, and those places are defined as interesting ones to the user. The result was defining places of interests from mobility data by analysing place-related patterns of events and movements. However, the work presented in [10] relied on the data collected by many users in the area, while our work is designed for detecting and logging personal preferences, so activities in our work represent the personal life experiences of the user. In addition, no prototype application was done in [10] so the user cannot review and save the detected places and events for later retrieval.

Another work was presented by Wolf et al. [22] that relied on the loss of the GPS data as indication of buildings. Losing GPS signals within a given Radius on three different occasions is interpreted by the system as a building has been entered. This work identified only buildings and provided no detection for outdoor places or any activities.

The effect of using locations and images on memory recall has been tested by Kalnikaite et al. [23]. In their work, SenseCam images are associated with locations based on time and then presented to the user through an application. However, images are associated without the use of any particular clustering techniques. Thus if a SenseCam timestamp falls within 50 seconds of a GPS timestamp, that image and the GPS point will be paired together. Another application that presents groups of images on a map based on their locations has been created by Toyama et al. [24]. All the images are tagged by the location data and stored in a database, and then the application groups the images and shows them on the map based on the tagged location. Locations can be cities, streets, or user-defined places. This application lacks the automatic detection of important places as it relies mostly on the tagged data of the images.

In summary, the work presented in this paper extends the previously mentioned works by presenting techniques that cluster location data and then grouping a sequence of images based on the clustering results. The authors combine recognition of places with inference of activities relying solely on time-stamped location data. Context and Content data are also combined and visualized through a prototype application, so the user can mark places and activities that happened at interesting stops to retrieve them later on. In addition, having a stream of images can help the user, when reviewing through the proposed application, in naming what activity happened in the place.

\section{Discussion}

This section discusses the results of the efforts with respect to the research questions.

The first addressed question is: "How can places of importance be recognized and activities be inferred based on location data and time?"

Places can be recognized relying solely on time-stamped location data using the DBSCAN algorithm. DBSCAN aggregates GPS points into clusters based on the density of points. The authors calibrated the density-based algorithm based on data collected by three users over a period of six months. The best parameter values for DBSCAN that result in fewer numbers of place recognition errors are 12.8 meters for the Radius and 3 points for MinPts. OPTICS algorithm is also used to ensure that the chosen parameters values for DBSCAN provide stable clustering results. The DBSCAN algorithm results in clusters that represent places visited by the user. After the clusters are identified, the 
system constructs the convex hull to estimate the geographical boundaries of the recognized places.

Activities are inferred based on the known places and the essential places that are defined in the previous step. An activity is represented by a set of GPS points which belong to the same place and which are sequential in time. The system searches within the defined place clusters and splits them into sub-clusters that do not overlap in time. Each subcluster represents an activity that happened in a certain place at a certain time. The timeframe of each activity is the time between the earliest point and the latest one within the sub-cluster. A cluster, which represents a place, might be divided into several subclusters, which represent activities happened in the same place at different time.

The second addressed question is: "How can structured lifelogs be presented so the user can review and retrieve the life experiences?"

The lifelogs, which are structured based on places and activities, are presented through a prototype application that answers the following questions:

- When did the activity take place? The timeframe of the activity is presented based on the identified corresponding time-stamped GPS points.

- Where was the activity? The place where the activity happened is presented on the map based on a convex hull of the corresponding GPS points.

- What was the user doing? The presentation of the activity is based on the autocaptured images, which were taken at the time of the activity.

A SenseCam can be used to capture images automatically while a mobile device can collect GPS points during the day of the person. The system transfers all the logs when connecting those portable devices to a computer, and then defines places and activities based on the GPS data. SenseCam images are then associated with those places and activities based on time and presented to the user for reviewing and adjustment. Adding SenseCam images, as content, to the clustering results helps the user in naming places and activities when reviewing.

If the user confirms a cluster as a place, the coordinates that correspond to this place are saved and the place will be known and detected automatically next visit. Therefore the system can improve its knowledge about the user's preferable places. Saving activities will just save the data and make it available for later retrieval. The system thus presents the structured lifelogs as places and activities associated with SenseCam images. The system helps the user to retrieve or share previous moments in life based on places or time. For example, the user can review all the activities that happened in a certain place, such as the university, or at a certain time, such as the New Year eve.

\section{Conclusion and Future Work}

This article presented a novel approach that relies on location data and images to organize the lifelogs of someone's life. Location data provides a context source that can be used to recognize places and infer activities. Images, as content data, can be then associated with those recognized places and inferred activities, and be presented to the user for reviewing and adjustment. The introduced prototype system structures and presents lifelogs based on places, activities and images that can be available for later retrieval. The system therefore provides a digital tool for people to reminisce and share their life. 
The next stage of our work is improving the inference of activities within the lifelogging system using the same set of devices. Sensor-readings in SenseCam can be used with image processing techniques to better reason about daily activities. This will also help the system distinguishing between different activities that usually happen in the same place, which will improve the activity inference task.

\section{References}

[1] Byrne D., Lavelle B., Doherty A. R., Jones G.J.F., \& Smeaton A.F., 2007. Using Bluetooth \& GPS Metadata to Measure Event Similarity in SenseCam Images. Centre for Digital Video Processing (CDVP) \& Adaptive Information Cluster (AIC), Dublin City University, Dublin 9, Ireland.

[2] Doherty A.R., 2009. Providing effective memory retrieval cues through automatic structuring and augmentation of a lifelog of images. PhD thesis, Dublin City University.

[3] Kikhia B., Hallberg J., Bengtsson J.E., Sävenstedt S. and Synnes K., 2010. Building digital life stories for memory support. Int. J. Computers in Healthcare, Vol. 1, No. 2, pp.161-176.

[4] Anind K. Dey and Gregory D. Abowd, 2000. Towards a Better Understanding of Context and Context-Awareness, 2000. In: Workshop on The What, Who, Where, When, and How of Context-Awareness, as part of the 2000 Conference on Human Factors in Computing Systems (CHI 2000), The Hague, The Netherlands.

[5] Frohlich D., Kuchinsky A., Pering C., Don A., and Ariss S., 2002. Requirements for photoware. In Proc. of CSCW'02, ACM Press, New Orleans, Louisiana, USA.

[6] Chalfen R., 1997. Family photography: One album is worth a 1000 lies, In D. M. Neuwman, ed., Sociology: Exploring the architecture of everyday life, CA.: Pine Forge Press, pp. 269-278.

[7] Ester M., Kriegel H.-P., Sander J., and Xu X., 1996. A density-based algorithm for discovering clusters in large spatial databases with noise. In Proc KDD. AAAI Press, Volume: 96, Pages: 226-231. ISBN: 1577350049.

[8] Ankerst M., Breunig M.M., Kriegel H., and Sander J., 1999. OPTICS: ordering points to identify the clustering structure. In Proceedings of the 1999 ACM SIGMOD international conference on Management of data (SIGMOD '99). ACM, New York, NY, USA, 49-60.

[9] Palma A. T., Bogorny V., Kuijpers B., Alvares L.O., 2008. A Clustering-based Approach for Discovering Interesting Places in Trajectories. In: 23rd Annual Symposium on Applied Computing, (ACM-SAC'08), Fortaleza, Ceara, 16-20 March (2008) Brazil. pp. 863-868.

[10] Andrienko G., Andrienko N., Hurter C., Rinzivillo S., Wrobel S., 2011. From Movement Tracks through Events to Places: Extracting and Characterizing Significant Places from Mobility Data. IEEE Visual Analytics Science and Technology (VAST 2011) Proceedings, IEEE Computer Society Press, pp.161170.

[11] Cormen T.H., Leiserson C.E., Rivest R.L., Stein C., 2009. Introduction to Algorithms (3rd ed.). MIT Press. ISBN 0-262-03384-4. 
[12] Hall M., Frank E., Holmes G., Pfahringer B., Reutemann P., and Witten I. H., 2009. The WEKA data mining software: an update, SIGKDD Explor. Newsl., vol. 11 , no. 1 , pp. 10-18, Nov.

[13] Kikhia B., Bengtsson J.E., Synnes K., Ul Hussain Sani Z., and Hallberg J., 2010. Creating Digital Life Stories through Activity Recognition with Image Filtering. In the proceeding of the 8th International Conference on Smart Homes and Health Telematics (ICOST), Seoul, South Korea. Pages 203-210.

[14] Gemmell J., Williams L., Wood K., Bell G. and Lueder R., 2004. Passive Capture and Ensuing Issues for a Personal Lifetime Store, Proceedings of The First ACM Workshop on Continuous Archival and Retrieval of Personal Experiences (CARPE '04), New York, NY, USA, pp. 48-55.

[15] Byrne D., Doherty A.R., Snoek C.G.M., Jones G.F., and Smeaton A.F., 2008. Validating the Detection of Everyday Concepts in Visual Lifelogs. SAMT 2008 3rd International Conference on Semantic and Digital Media Technologies, Koblenz, Germany, 3-5 D.

[16] Doherty A.R, Ó Conaire C, Blighe M, Smeaton A.F, and O'Connor N., 2008. Combining Image Descriptors to Effectively Retrieve Events from Visual Lifelogs. MIR 2008 - ACM International Conference on Multimedia Information Retrieval, Vancouver, Canada, 30-31.

[17] Hodges S., Williams L., Berry E., Izadi S., Srinivasan J., Butler A., Smyth G., Kapur N., and Wood K., 2006. Sensecam: A retrospective memory aid. In UbiComp: 8th International Conference on Ubiquitous Computing, volume 4602 of LNCS.

[18] Wilkin G.A., XiuzhenH., 2007. K-Means Clustering Algorithms: Implementation and Comparison. Second International Multi-Symposiums on Computer and Computational Sciences (imsccs), pp.133-136.

[19] Zhou C., Frankowski D., Ludford P., Shekhar S., and Terveen L., 2004. Discovering personal gazetteers: An interactive clustering approach. In Proc. ACMGIS, pages 266-273.

[20] Ashbrook D., Starner S., 2002. Learning Significant Locations and Predicting User Movement with GPS. Proceedings of the 6th IEEE International Symposium on Wearable Computers, p.101.

[21] Alvares L. O., Bogorny V., Kuijpers B., de Macedo J. A. F. , Moelans B., and Vaisman A., 2007. A model for enriching trajectories with semantic geographical information. In GIS'07: Proceedings of the 15th Annual ACM International Symposium on Advances in Geographic Information Systems, New York, NY, USA, ACM Press.

[22] Wolf J., Guensler R., and Bachman W., 2001. Elimination of the travel diary: An experiment to derive trip purpose from GPS travel data. In: Notes from Transportation Research Board, 80th annual meeting, Washington, D.C.

[23] Kalnikaite V., Sellen A., Whittaker S. and Kirk D., 2010. Now Let Me See Where I Was: Understanding How Lifelogs Mediate Memory. CHI 2010, ACM Press, Atlanta, GA, USA.

[24] Toyama K., Logan R., Roseway A., and Anandan P., 2003. Geographic Location Tags on Digital Images. In the Proceedings of the eleventh ACM international conference on Multimedia, Berkeley, California, November 2003, 1-58113-722-2. 